\title{
Article \\ Efficient Wireless Power Transfer via Magnetic Resonance Coupling Using Automated Impedance Matching Circuit
}

\author{
Esraa Mousa Ali ${ }^{1}$, Mohammad Alibakhshikenari ${ }^{2, *}{ }^{\infty}$, Bal S. Virdee ${ }^{3}$, Mohammad Soruri ${ }^{4}$ and Ernesto Limiti ${ }^{5}(\mathbb{C}$ \\ 1 Faculty of Aviation Sciences, Amman Arab University, Amman 11953, Jordan; Esraa_ali@aau.edu.jo \\ 2 Department of Signal Theory and Communications, Universidad Carlos III de Madrid, Leganés, \\ 28911 Madrid, Spain \\ 3 Center for Communications Technology, London Metropolitan University, London N7 8DB, UK; \\ b.virdee@londonmet.ac.uk \\ 4 Faculty of Ferdows Technical, University of Birjand, 9717434765 Birjand, Iran; mohamad.soruri@birjand.ac.ir \\ 5 Electronic Engineering Department, University of Rome “Tor Vergata”, Via del Politecnico 1, \\ 00133 Rome, Italy; limiti@ing.uniroma2.it \\ * Correspondence: mohammad.alibakhshikenari@uc3m.es
}

check for updates

Citation: Ali, E.M.;

Alibakhshikenari, M.; Virdee, B.S.; Soruri, M.; Limiti, E. Efficient

Wireless Power Transfer via Magnetic Resonance Coupling Using Automated Impedance Matching Circuit. Electronics 2021, 10, 2779. https://doi.org/10.3390/

electronics10222779

Academic Editor: Fabian Khateb

Received: 21 October 2021

Accepted: 10 November 2021

Published: 13 November 2021

Publisher's Note: MDPI stays neutral with regard to jurisdictional claims in published maps and institutional affiliations.

Copyright: (c) 2021 by the authors. Licensee MDPI, Basel, Switzerland. This article is an open access article distributed under the terms and conditions of the Creative Commons Attribution (CC BY) license (https:/ / creativecommons.org/licenses/by/ $4.0 /)$.

\begin{abstract}
In this paper, an automated impedance matching circuit is proposed to match the impedance of the transmit and receive resonators for optimum wireless power transfer (WPT). This is achieved using a 2D open-circuited spiral antenna with magnetic resonance coupling in the low-frequency ISM band at $13.56 \mathrm{MHz}$. The proposed WPT can be adopted for a wide range of commercial applications, from electric vehicles to consumer electronics, such as tablets and smartphones. The results confirm a power transfer efficiency between the transmit and receive resonant circuits of $92 \%$, with this efficiency being sensitive to the degree of coupling between the coupled pair of resonators.
\end{abstract}

Keywords: wireless power transfer (WPT); automated impedance matching circuit; magnetic resonant coupling

\section{Introduction}

Traditional vehicles based on fossil fuels are beginning to be replaced with electric vehicles (EV), to circumvent climate change and reduce carbon footprints [1-3]. Although EVs have been growing in popularity, rapid market growth is hampered by their limited driving range. This is because of the limited number of charging stations and the charging being time consuming when compared to petrol-powered cars. Furthermore, EVs must be charged more frequently than a fossil fuel vehicle, because the state-of-the-art battery technology is not capable of storing a large amount of electric charge. The limited energy density storage capacity of batteries necessitates frequent charging of EVs. Charging EVs is inconvenient, as the vehicle must be plugged into a charging station for at least $35 \mathrm{~min}$, which handicaps EVs. The repair and maintenance of EVs outside of the manufacturer is increasing. People in the motor vehicle repair and recovery industry are exposed to the hazard of the high voltage in EVs. Voltages present in EVs are significantly higher (typically 650 Volts dc) than those used in other vehicles (12/24 Volts dc). In dry conditions, accidental contact with parts that are live at voltages above 110 Volts dc can be fatal. For EVs, dc voltages between 60 and 1500 Volts are referred to as 'high voltage'. One solution to overcome this kind of hazard is to employ near-field wireless power transfer (WPT). Apart from making the charging of EVs more convenient, this charging method is safer for EV repair and maintenance workers and the general public. Indeed, since it does not involve the physical connection of any parts to the EV, it avoids any potential danger of electrocution, especially under raining conditions or when a power cable is damaged.

WPT technology is used currently to charge the batteries of some small and large devices, including mobile phones [4], medical implants [5], and, to a limited extent, EVs [6]. 
Currently, the limiting factor in WPT technology is its low efficiency. Therefore, to achieve effective power transfer the system must be highly efficient and must be capable of transferring power through large air gaps. From a technological viewpoint, WPT can be achieved using several techniques, including electromagnetic induction and microwave power transfer. The main shortcoming of these technologies are short-range (a few centimeters) and low efficiency [7]. Moreover, the efficiency of electromagnetic induction declines substantially when there is misalignment between the transmitter and receiver coils. It has been shown that a higher efficient can be achieved using magnetic resonance coupling. This technology can provide an efficiency of approximately $90 \%$ within one meter [8,9]. However, the resonance frequencies of the transmit/receive resonators change as the gap is varied. This means that for such a system to be practically viable, resonance parity must be maintained independently of the gap, to maintain the efficiency performance of the WPT system. In this context, various studies have shown that resonance conditions can be maintained using techniques such as (i) impedance matching [7], (ii) frequency matching [10], and (iii) manipulation of coupling [11]. For practical application purposes, such resonance matching systems need to be automated. Although frequency matching and coupling manipulation are not suitable for automation, impedance matching systems are viable, since they do not involve any moving parts.

More recently various WPT technologies have been reported [12-17]. In [12], a wireless power supply for an on-board charger used a bifurcation approach, where the secondaryvoltage controller based on primary-side control provides constant-voltage gain under various loads and coupling coefficients. Constant voltage gain was shown to be achieved in both the coreless power pad and the pad with ferrites, providing flexibility of coupling coefficient and inductance variations. Experimental results confirmed that the system reported has a maximum efficiency of $90 \%$. The wireless power transfer via magnetic resonance coupling in [13] offers the prospect of autonomously charging connected devices. This was achieved using a surface routing approach, which was found to be more efficient than linear routing. The challenge of cross-coupled relays was overcome here by conditioning the resonators' current by appending additional loading conditions to the relays. Simulations showed that the maximum efficiency of the proposed method is $75 \%$. In [14], a WPT concept based on the eddy-current testing and evaluation method was presented. This approach involved multiple resonance frequencies as features for crack detection and characterization. As a result, the selection of specific features for the best sample and crack specifications was permitted, by using multiple resonance frequencies. A wireless power transfer system for multiple loads was reported in [15]. Multiple coils were used as power repeaters to enhance the power transfer capability. A inductor-capacitor-capacitor compensation method was used for each repeater coil in the system, which ensures loadindependent currents flow through the loads. Bipolar coils are used for both the repeater coil and compensation coil. The undesirable coupling coefficient between the repeater coil and its compensation coil is eliminated by placing them perpendicularly. A compensation coil is also used to transfer energy to the next repeater coil via magnetic coupling. The maximum efficiency achieved was $86.6 \%$. A multi-load WPT system using repeater units containing two repeater coils was reported in [16]. This system uses a series-parallel-series compensation topology for each repeater unit. The load is connected in series with the receiving coil in each repeater unit. With the proposed system structure and compensation topology, constant load currents can be obtained for all the loads. The maximum efficiency achieved by this system was $82.5 \%$. A method for achieving simultaneous WPT and fullduplex communication with a pair of coupling coils was reported in [17]. Power transfer is achieved using a double-side LCC compensation topology. The maximum power transfer efficiency realized was $86 \%$.

In this paper, we propose a high efficiency wireless power transfer system, using a magnetic resonance coupling system. The resonance frequency of the proposed system frequency is impedance matched to the energy source at $13.56 \mathrm{MHz}$, which is within the industrial, scientific, and medical (ISM) band. A feasibility study showed that by 
employing an automatic impedance matching circuit, the efficiency of the WPT system can be significantly improved.

\section{Magnetic Resonance Coupling}

Magnetic resonant coupling theory is well established and may also be found in mode coupling methods [8]. Resonator coupling was analyzed using equivalent circuits in $[7,9]$. The open-circuit spiral antenna shown in Figure 1 was used for magnetic resonant coupling, to achieve wireless power transfer. The antenna was fabricated using standard manufacturing techniques on Rogers $\mathrm{RO} 4003$ dielectric substrate, with a thickness of $0.5 \mathrm{~mm}$, a dielectric constant $\left(\varepsilon_{r}\right)$ of 3.38 , and a loss-tangent $(\delta)$ of 0.0027 .

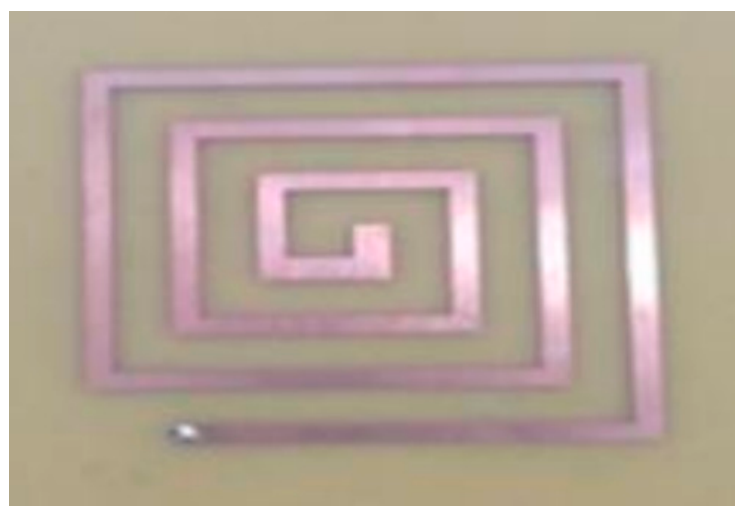

Figure 1. Prototype spiral antenna for wireless power transfer.

\subsection{Equivalent Circuit of Magnetic Resonant Coupling}

Figure 2 shows an equivalent circuit model illustrating how WPT is achieved using magnetic resonant coupling. This is realized by means of magnetic coupling via $L C$ resonance circuits. The magnetic coupling is represented by the mutual inductance $\left(L_{m}\right)$. The characteristic impedance of the source is $Z_{S}$, and $Z_{L}$ is the impedance of the load. The loss in the circuit is represented by resistance $(R)$ and the characteristic impedance is $Z_{0}=50 \Omega$.

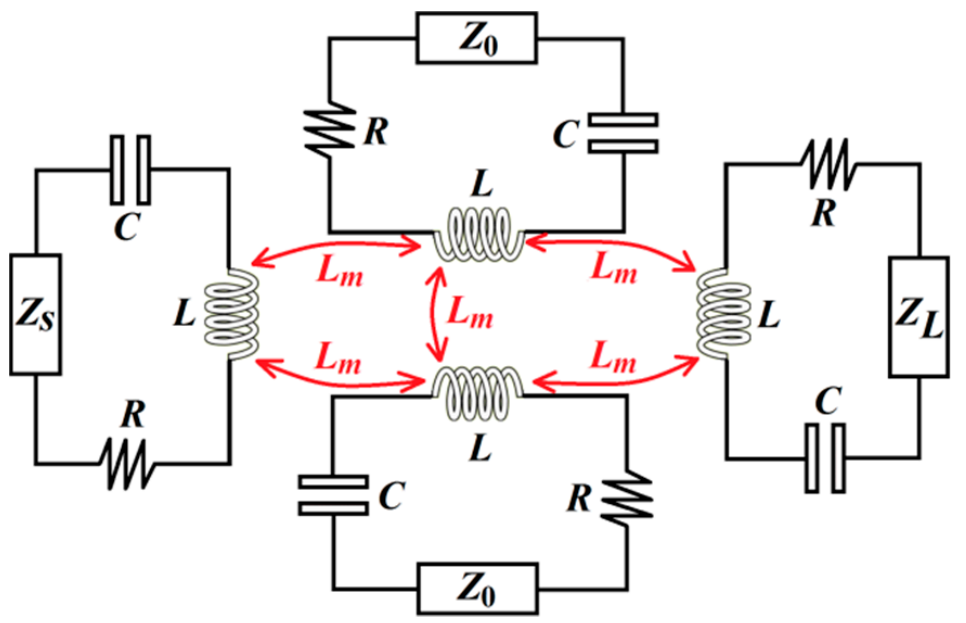

Figure 2. Simplified equivalent circuit of wireless power transfer system via magnetic resonance coupling.

The equivalent circuit in Figure 2 can be analyzed by considering the interaction between only two resonators. Figure 3a shows the equivalent lumped-element circuit model for just the two magnetically coupled resonator structures, where $L$ and $C$ are the self- 
inductance and self-capacitance, and $L_{m}$ represents the mutual inductance. The coupling equations describing the two-port network at reference planes $T 1-T^{\prime} 1$ and $T 2-T^{\prime} 2$ are

$$
\begin{aligned}
& v_{1}=j \omega L i_{1}+j \omega L_{m} i_{2} \\
& v_{2}=j \omega L i_{2}+j \omega L_{m} i_{1}
\end{aligned}
$$

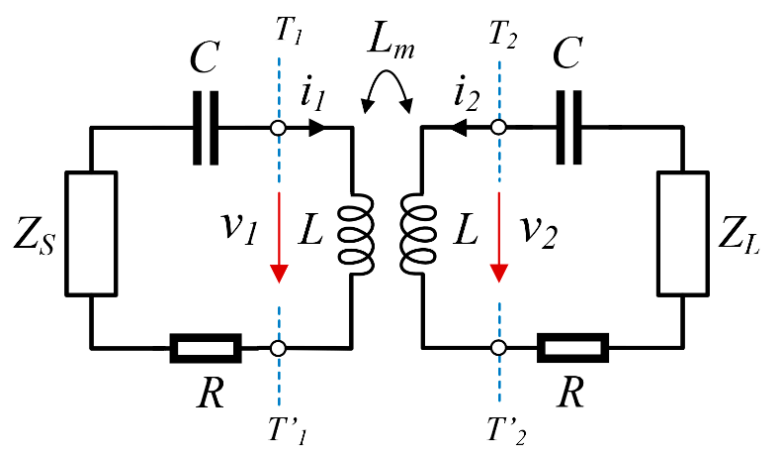

(a)

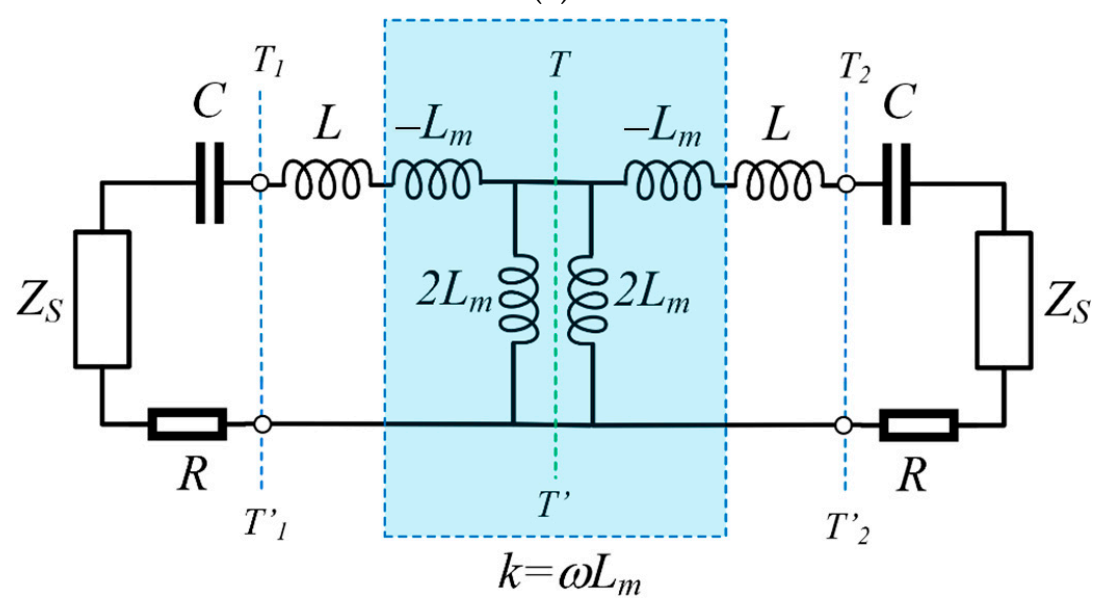

(b)

Figure 3. (a) Coupled resonator circuit with magnetic coupling, and (b) an alternative form of the equivalent circuit with an impedance inverter $k=\omega L_{m}$ to represent the coupling.

The second terms on the right hand side of Equations (1) and (2) are the induced voltages resulting from the current flow in loops 2 and 1, respectively. The loop currents in Figure 3a flow in the opposite directions, so that the voltage drops, due to the mutual inductance. An alternative representation of an equivalent circuit is shown in Figure 3b. The magnetic coupling between the two resonant loops is represented by an impedance inverter $k=\omega L_{m}$. If the symmetry plane $T-T^{\prime}$ in Figure $3 \mathrm{~b}$ is replaced by an electric wall, the resultant resonant frequency of the resonant circuit can be shown to be [18]

$$
f_{e}=\frac{1}{2 \pi \sqrt{\left(L-L_{m}\right) C}}
$$

If a magnetic wall (or an open circuit) replaces the symmetry plane in Figure $3 b$, the resultant single resonant circuit has a resonant frequency

$$
f_{m}=\frac{1}{2 \pi \sqrt{\left(L+L_{m}\right) C}}
$$


The overall resonant frequency is

$$
f_{o}=\sqrt{f_{e} f_{m}}
$$

Equations (3) and (4) can be used to find the magnetic coupling coefficient, which is defined by [18]

$$
k_{m}=\frac{f_{e}^{2}-f_{m}^{2}}{f_{e}^{2}+f_{m}^{2}}=\frac{L_{m}}{L}
$$

In the case of the four magnetically coupled identical resonators in Figure 2, the overall resonant frequency and coupling coefficient between the neighboring resonators are defined by Equations (5) and (6), respectively.

The electrical and physical parameters of the $L C$ resonator used in this work are listed in Table 1. The electrical parameters were measured at $13.56 \mathrm{MHz}$ using a network analyzer. The resonator used here was made by stacking together four open-circuit spiral antennas. The associated capacitance resulted from the gap between the stacked antenna layers.

Table 1. Optimized Spiral Coil Resonator Parameters.

\begin{tabular}{cc}
\hline Antenna Type & Open-Circuit Rectangular Spiral Coil \\
\hline Number of spiral turns & 4 \\
Width of vertical spiral coil & $3 \mathrm{~mm}$ \\
Width of horizontal spiral coil & $2 \mathrm{~mm}$ \\
Vertical gap between the spiral coils & $5.5 \mathrm{~mm}$ \\
Horizontal gap between the spiral coils & $4 \mathrm{~mm}$ \\
Inductance, $L$ & $7100 \mathrm{nH}$ \\
Capacitance, $C$ & $18.4 \mathrm{pF}$ \\
Resistance, $R$ & $2.3 \Omega$ \\
$Q$ value @ $13.56 \mathrm{MHz}$ & 460 \\
\hline
\end{tabular}

The reflection ratio $\left(\eta_{11}\right)$ and the transmission ratio $\left(\eta_{21}\right)$ are defined in [1] and shown in Equations (7) and (8), where $S_{11}$ and $S_{21}$ represent the reflection and transmission coefficients, respectively.

$$
\begin{gathered}
\eta_{11}=\left|S_{11}\right|^{2} \times 100 \% \\
\eta_{21}=\left|S_{21}\right|^{2} \times 100 \% \\
S_{21}(\omega)=\frac{j L_{m} Z_{0} \omega L / C}{\left(\omega L_{m}\right)^{2}+j\left[\left(\omega L+\frac{1}{\omega C}\right)+Z_{0}\right]^{2}}
\end{gathered}
$$

\subsection{Frequency Characteristics of Magnetic Resonant Coupling}

The gap between the transmit and receive resonant pair will affect the coupling coefficient and, therefore, will change the impedance of the system. The consequence of this is a change in the system's resonance frequency and, hence, the efficiency of the power transfer. Figure 4 shows the measured frequency characteristics of $\eta_{11}$ and $\eta_{21}$ at $15 \mathrm{MHz}$. The horizontal $\left(g_{h}\right)$ and vertical air gap $\left(g_{v}\right)$ between the transmit and receive resonators were altered between $4 \mathrm{~mm}$ to $20 \mathrm{~mm}$, and from $5.5 \mathrm{~mm}$ to $27.5 \mathrm{~mm}$, respectively. The results show that when the gap is relatively small the strong coupling creates four distinct resonance ripples, which contribute towards optimizing the WPT efficiency. As the gap is made larger, the resonance ripples move closer to each other and eventually coalesce, when $g_{v}=16 \mathrm{~mm}$ and $g_{h}=22 \mathrm{~mm}$. Any further increase in gap reduces the efficiency of the wireless power transfer. The relationship between $k$ and $g$ is shown in Figure 5. 


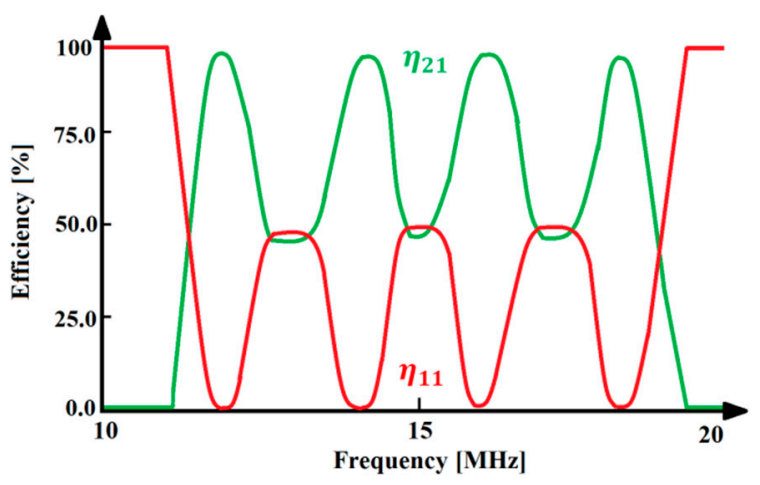

(a)

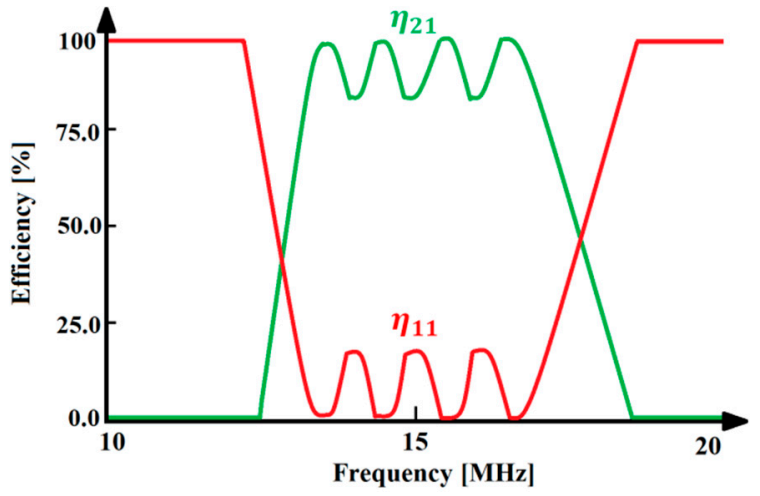

(b)

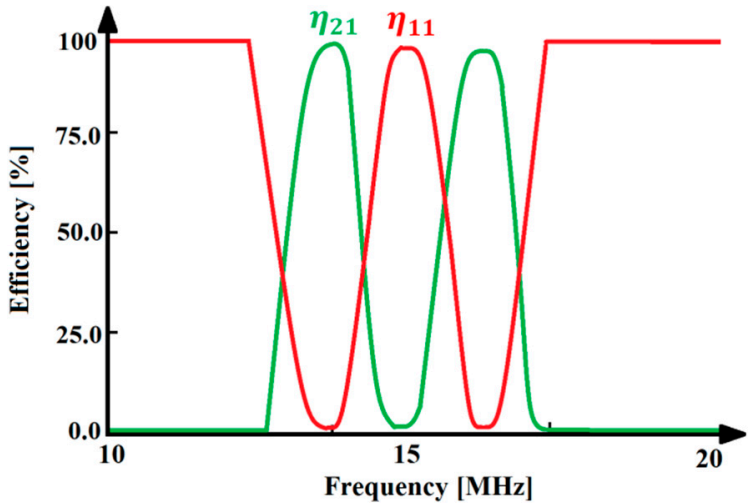

(c)

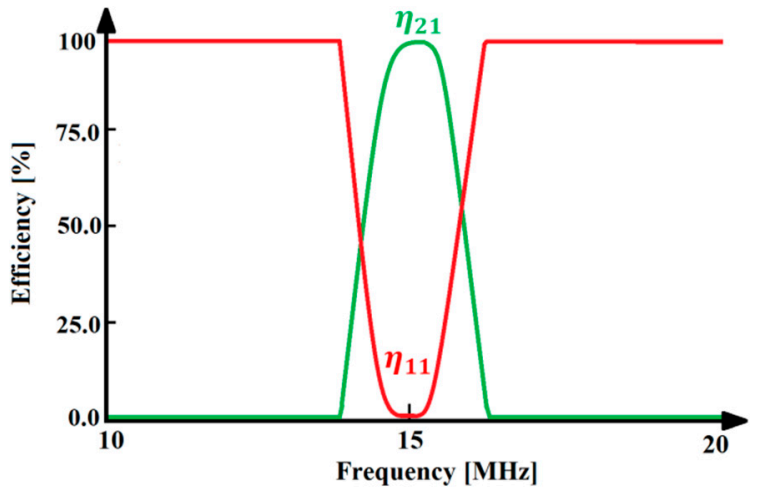

(d)

Figure 4. Cont. 


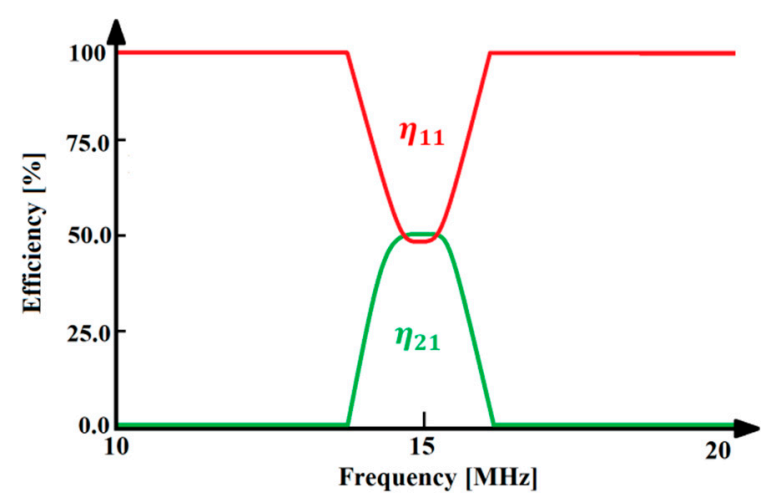

(e)

Figure 4. Experimental results of WPT efficiency versus frequency with different air gaps. (a) $g_{v}=4 \mathrm{~mm}$ and $g_{h}=5.5 \mathrm{~mm}$. (b) $g_{v}=8 \mathrm{~mm}$ and $g_{h}=11 \mathrm{~mm}$. (c) $g_{v}=12 \mathrm{~mm}$ and $g_{h}=16.5 \mathrm{~m}$. (d) $g_{v}=16 \mathrm{~mm}$ and $g_{h}=22 \mathrm{~mm}$. (e) $g_{v}=20 \mathrm{~mm}$ and $g_{h}=27.5 \mathrm{~mm}$.

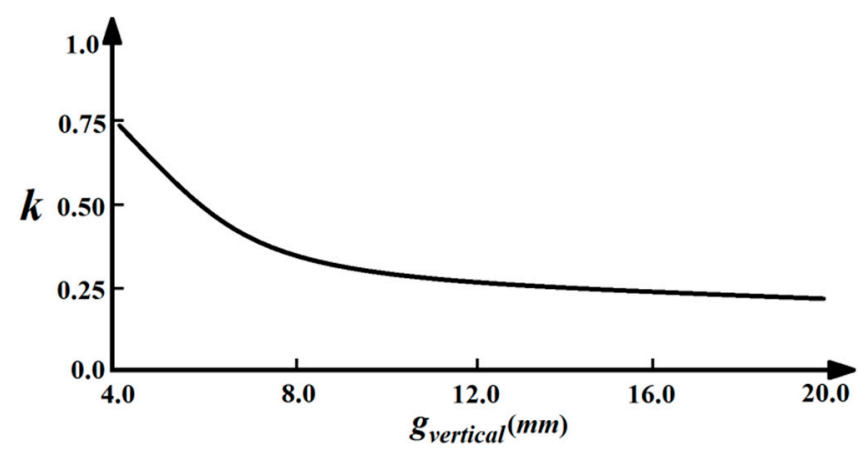

(a)

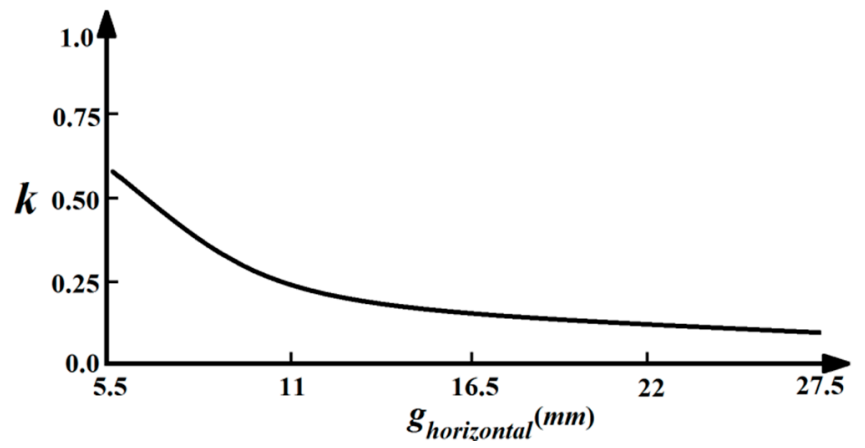

(b)

Figure 5. Coupling coefficient ( $k$ ) versus air gap (g). (a) $4 \leq g_{v} \leq 20$. (b) $5.5 \leq g_{h} \leq 27.5$.

\section{Effect of Impedance Matching Circuit on Magnetic Resonant Coupling}

The proposed automated impedance matching circuit (IMC) used to maximize the power transfer between the transmit and receive antennas is illustrated in Figure 6. The IMC is used to equalize the impedance of the resonant transmit and receive antennas. It consists of a tunable matching network that generates the desired impedance transformation based on the output of an impedance sensor, which comprises a directional coupler and detector diode for mismatch measurement; a switch; a switch timing generator; and a time constant generator. The varactor diode in the matching circuit provides electronically controllable capacitance. The system uses the magnitude of the return-loss measured at the spiral antenna to determine the degree of mismatch. Information on the phase of the return-loss is also used to accurately minimize the degree of mismatch. The proposed system uses a test signal to determine whether the mismatch increases or decreases. Knowledge of this is used to accurately control the capacitance in the matching circuit. This is achieved 
by turning the switch 'on' to increase the control voltage to the variable varactor $(C)$. If the mismatch increases, the system acknowledges this by turning the switch 'off'. If the mismatch reduces, the system acknowledges this by keeping the switch 'on'. The voltage is maintained using sample-and-hold $(\mathrm{S} / \mathrm{H})$ circuitry. Power transfer is achieved via magnetic resonance coupling between the resonators. The received power is rectified, and the electrical charge is stored in the battery, which comprises electric double-layer capacitors (EDLCs). Impedance matching is autonomously controlled by an algorithm. Hence, adaptive impedance matching is achieved in real-time.
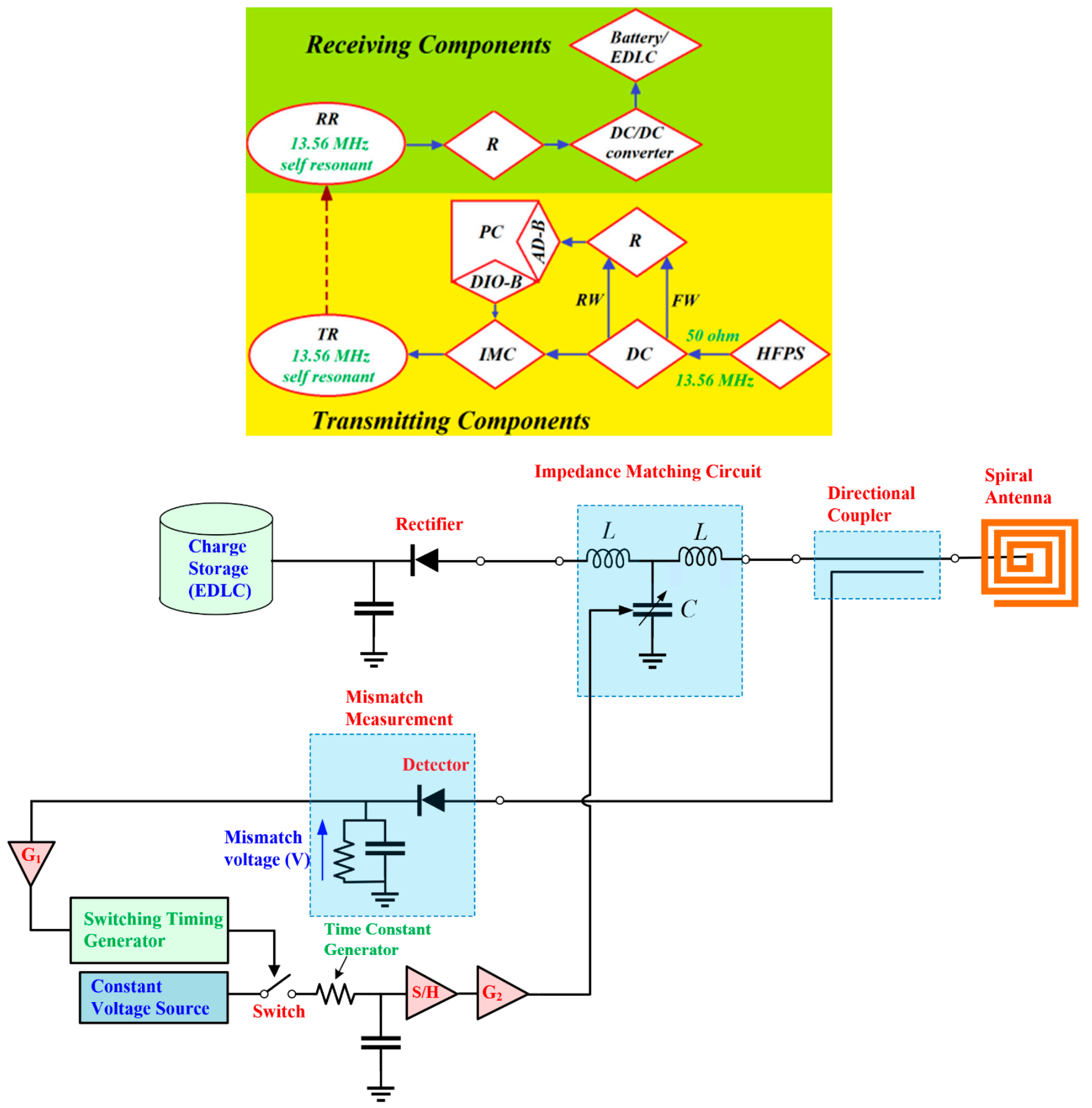

Figure 6. Proposed wireless power transfer system with an automated impedance matching circuit (IMC). HFPS, DC, FW, RW, R, DIO-B, AD-B, PC, TR, RR, DC/DC, and EDLC signify high frequency power source, directional coupler, forwarded wave, reflected wave, rectifier, digital input-output board, AD board, personal computer, transmitting resonator, receiving resonator, direct current/direct current, and electric double-layer capacitor. 
The efficiency of the IMC on the magnetic resonance coupling for wireless power transfer was measured at $13.56 \mathrm{MHz}$. Figure 7a shows that before matching, the system resonates at $13 \mathrm{MHz}$ and $14.3 \mathrm{MHz}$. The efficiency at these two frequencies is $72 \%$. After matching, the system resonates at $13.56 \mathrm{MHz}$ and the efficiency increased to $92 \%$, which is higher than the other approaches recently reported in [12-17]. With the application of IMC the efficiency of the WPT between the transmit resonant circuit and the receive resonant circuit was enhanced by $22 \%$ at $13.56 \mathrm{MHz}$. When the coupling coefficient $(k)$ was reduced from 0.64 to 0.48 , as shown in Figure $7 b, c$, the corresponding decrease in efficiency was from $82 \%$ to $63 \%$.

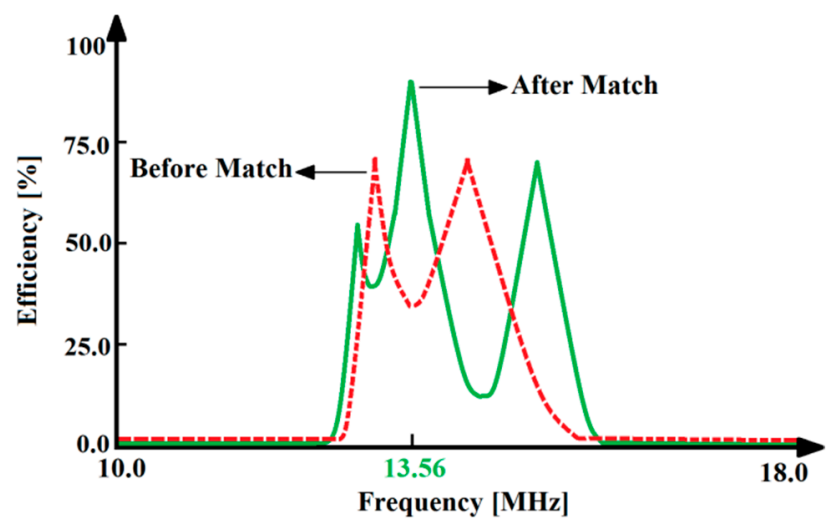

(a)

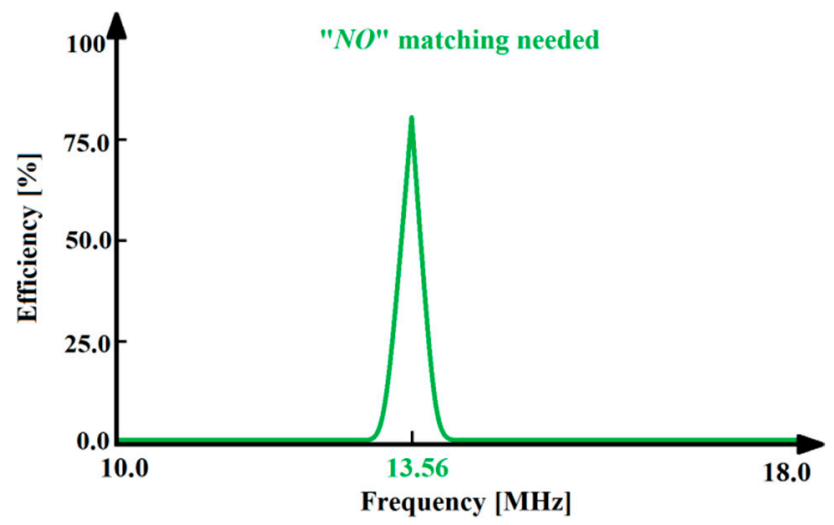

(b)

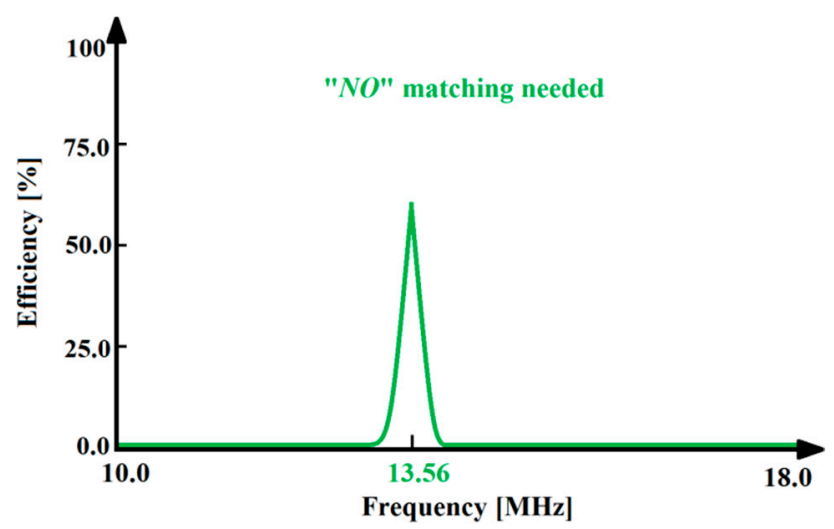

(c)

Figure 7. Frequency characteristics of the system $\left(\eta_{21}\right)$ before and after matching. (a) $k=0.74$. (b) $k=0.64$. (c) $k=0.48$. 


\section{Conclusions}

Measurement results show that the proposed automatic impedance matching circuit (IMC), when applied to magnetic resonance coupling, increased the efficiency of the wireless power transfer between the transmit resonant circuit and the receive resonant circuit at $13.56 \mathrm{MHz}$ by $22 \%$. The proposed IMC automation can be easily implemented to make power transfer systems robust to changes in the air gap between the transmit and receive resonators. However, the efficiency of the proposed approach is sensitive to the degree of coupling between the coupled pairs of resonators.

Author Contributions: Conceptualization, E.M.A., M.A., B.S.V., M.S. and E.L.; methodology, E.M.A., M.A., B.S.V., M.S. and E.L.; software E.M.A., M.A., B.S.V. and M.S.; validation, E.M.A., M.A., B.S.V., M.S. and E.L.; formal analysis, E.M.A., M.A., B.S.V. and M.S.; investigation, E.M.A., M.A., B.S.V., M.S. and E.L.; resources, E.M.A., M.A., B.S.V. and M.S.; data curation, E.M.A., M.A., B.S.V. and M.S.; writing-original draft preparation, E.M.A., M.A. and M.S.; writing-review and editing, E.M.A., M.A., B.S.V., M.S. and E.L.; visualization, E.M.A., M.A., B.S.V., M.S. and E.L.; supervision, M.A. and E.L.; project administration, E.M.A., M.A., B.S.V. and E.L.; funding acquisition, M.A. and E.L. All authors have read and agreed to the published version of the manuscript.

Funding: This project received funding from the Universidad Carlos III de Madrid and the European Union's Horizon 2020 research and innovation programme under the Marie Sklodowska-Curie Grant 801538.

Institutional Review Board Statement: Not applicable.

Informed Consent Statement: Not applicable.

Data Availability Statement: Data are contained within the article.

Acknowledgments: The authors sincerely appreciate the funding support from Universidad Carlos III de Madrid and the European Union's Horizon 2020 research and innovation programme under the Marie Sklodowska-Curie Grant 801538.

Conflicts of Interest: The authors declare no conflict of interest.

\section{References}

1. Beh, T.C.; Kato, M.; Imura, T.; Oh, S.; Hori, Y. Automated Impedance Matching System for Robust Wireless Power Transfer via Magnetic Resonance Coupling. IEEE Trans. Ind. Electron. 2012, 60, 3689-3698. [CrossRef]

2. Low, Z.N.; Chinga, R.A.; Tseng, R.; Lin, J. Design and test of a high-power high-efficiency loosely coupled planar wireless power transfer system. IEEE Trans. Ind. Electron. 2009, 56, 1801-1812.

3. Jeong, S.; Jang, Y.J.; Kum, D.; Lee, M.S. Charging Automation for Electric Vehicles: Is a Smaller Battery Good for the Wireless Charging Electric Vehicles? IEEE Trans. Autom. Sci. Eng. 2018, 16, 486-497. [CrossRef]

4. Jabbar, H.; Song, Y.S.; Jeong, T.T. RF energy harvesting system and circuits for charging of mobile devices. IEEE Trans. Consum. Electron. 2010, 56, 247-253. [CrossRef]

5. Shiba, K.; Morimasa, A.; Hirano, H. Design and Development of Low-Loss Transformer for Powering Small Implantable Medical Devices. IEEE Trans. Biomed. Circuits Syst. 2010, 4, 77-85. [CrossRef] [PubMed]

6. Madawala, U.K.; Thrimawithana, D.J. A Bidirectional Inductive Power Interface for Electric Vehicles in V2G Systems. IEEE Trans. Ind. Electron. 2011, 58, 4789-4796. [CrossRef]

7. Beh, T.C.; Imura, T.; Kato, M.; Hori, Y. Basic Study of Improving Efficiency of Wireless Power Transfer via Magnetic Resonance Coupling Based on Impedance Matching. In Proceedings of the 2010 IEEE International Symposium on Industrial Electronics, Bari, Italy, 4-7 July 2010; pp. 2011-2016. [CrossRef]

8. Karalis, A.; Joannopoulos, J.; Soljačić, M. Efficient wireless non-radiative mid-range energy transfer. Ann. Phys. 2008, 323, 34-48. [CrossRef]

9. Cheon, S.; Kim, Y.-H.; Kang, S.-Y.; Lee, M.L.; Lee, J.-M.; Zyung, T. Circuit-Model-Based Analysis of a Wireless Energy-Transfer System via Coupled Magnetic Resonances. IEEE Trans. Ind. Electron. 2011, 58, 2906-2914. [CrossRef]

10. Sample, A.; Meyer, D.T.; Smith, J.R. Analysis, Experimental Results, and Range Adaptation of Magnetically Coupled Resonators for Wireless Power Transfer. IEEE Trans. Ind. Electron. 2011, 58, 544-554. [CrossRef]

11. Duong, T.P.; Lee, J.-W. Experimental Results of High-Efficiency Resonant Coupling Wireless Power Transfer Using a Variable Coupling Method. IEEE Microw. Wirel. Compon. Lett. 2011, 21, 442-444. [CrossRef]

12. Hatchavanich, N.; Sangswang, A.; Konghirun, M. Secondary-Side Voltage Control via Primary-Side Controller for Wireless EV Chargers. IEEE Access 2020, 8, 203543-203554. [CrossRef] 
13. Morita, M.; Sasatani, T.; Takahashi, R.; Kawahara, Y. Surface Routing for Wireless Power Transfer Using 2-D Relay Resonator Arrays. IEEE Access 2021, 9, 133102-133110. [CrossRef]

14. Daura, L.U.; Tian, G.Y. Wireless Power Transfer Based Non-Destructive Evaluation of Cracks in Aluminum Material. IEEE Sensors J. 2019, 19, 10529-10536. [CrossRef]

15. Cheng, C.; Lu, F.; Zhou, Z.; Li, W.; Deng, Z.; Li, F.; Mi, C.C. A Load-Independent LCC-Compensated Wireless Power Transfer System for Multiple Loads with a Compact Coupler Design. IEEE Trans. Ind. Electron. 2020, 67, 4507-4515. [CrossRef]

16. Cheng, C.; Zhou, Z.; Li, W.; Zhu, C.; Deng, Z.; Mi, C.C. A Multi-Load Wireless Power Transfer System with Series-Parallel-Series Compensation. IEEE Trans. Power Electron. 2019, 34, 7126-7130. [CrossRef]

17. Fan, Y.; Sun, Y.; Dai, X.; Zuo, Z.; You, A. Simultaneous Wireless Power Transfer and Full-Duplex Communication with a Single Coupling Interface. IEEE Trans. Power Electron. 2021, 36, 6313-6322. [CrossRef]

18. Wong, J.-S.; Lancaster, M. Microstrip Filters for RF/Microwave Applications [Book Review]. IEEE Microw. Mag. 2002, 3, 62-65. [CrossRef] 